23

\title{
Неполяризующие интерференционные системы, содержащие металлические слои
}

\author{
(С) Фам Ван Хоа, Нго Тхай Фи, Л.А. Губанова \\ Университет ИТМО, \\ 197101 Санкт-Петербург, Россия \\ e-mail: phamhoa0303@mail.ru
}

Поступила в редакцию 11.01.2019 г.

В окончательной редакции 31.01.2019 г.

Принята к публикации 23.04.2019 г.

\begin{abstract}
Рассмотрена структура неполяризующих интерференционных систем, в состав которых входят слои, изготовленные из металлических материалов. Представлена методика поиска конструкций металлодиэлектрических интерференционных систем, обеспечивающих малое расхождение между спектральными характеристиками энергетического коэффициента отражения (пропускания) для $s$ - и $p$-поляризаций. Разработанные на основании представленной методики конструкции имеют достаточно простую структуру, обеспечивающую легкость их реализации. Анализ спектральных характеристик полученных конструкций показал, что в рассматриваемом диапазоне спектра $(460-580 \mathrm{~nm})$ разница между интегральными коэффициентами отражения для $s$ - и $p$-поляризаций не превышается $1 \%$.
\end{abstract}

Ключевые слова: поляризация, коэффициент отражения, угол падения.

DOI: $10.21883 /$ OS.2019.09.48212.2-19

\section{Введение}

Поляризация отраженного (прошедшего) излучения обычно возникает, когда интерференционные системы работают под некоторым наклонным углом. В этом случае коэффициент отражения (пропускания) состоит из двух компонент: $s$ - и $p$-поляризаций. Поляризация широко применяется в оптическом приборостроении для различных целей. Однако в ряде случаев явление поляризации оказывается негативным [1]. Это приводит к задаче поиска интерференционных систем, позволяющих получить незначительное расхождение между спектральными характеристики коэффициента отражения (пропускания) для $s$ - и $p$-поляризаций в широкой спектральной области.

В настоящее время существуют два пути решения задачи обеспечения минимального расхождения между спектральными характеристики коэффициента отражения (пропускания) для $s$ - и $p$-поляризаций. Каждое решение имеет свои достоинства и недостатки. Первое решение основано на построении методики поиска конструкций интерференционных систем, состоящих из только диэлектрических слоев. Данные методики представлены в ряде работ [2-5]. Достоинством конструкций, разработанных на основании этих методик, является обеспечение получения неполяризующих интерференционных систем без потери прошедшей интенсивности изза отсутствия в их составе поглощающих материалов, как металлы. Недостатком таких систем является то, что незначительное расхождение между двумя поляризациями наблюдается в узком спектральном диапазоне, обычно не более $100 \mathrm{~nm}$. Второе решение основывается на поиске конструкций интерференционных систем, в состав которых входят слои, изготовленные из металлических материалов. Благодаря тому, что металлические пленки обладают меньшим деполяризующим эффектом, чем диэлектрики, металлические интерференционные системы дают возможность обеспечения минимального расхождения между двумя поляризациями в довольно широкой спектральной области [6]. Основным недостатком таких систем является то, что использование металлических пленок часто сопровождается потерями интенсивности за счет поглощения в металлах и сложностями синтезирования структур покрытий в связи с тем, что показатели преломления и коэффициенты поглощения металлов обладают существенной дисперсией. В работе [7] представлено изменение коэффициента поглощения за счет внедрения в конструкцию металл/стекло дополнительного слоя, изготовленного из диэлектрического материала. Результаты исследования показали, что поглощение системы металл/стекло существенно уменьшается в присутствии диэлектрического слоя, расположенного между металлом и стеклом. Спектральные характеристики неполяризующих интерференционных систем, содержащих в конструкции металлические слои, были опубликованы в ряде работ [8-10]. Недостатком конструкций, представленных в этих работах, является то, что в их состав входит большое количество слоев. Причем толщины слоев не кратны между собой, что вызывает трудности в процессе изготовления неполяризующих интерференционных покрытий.

В настоящей работе рассматривается методика поиска конструкций неполяризующих интерференционных систем, изготовленных из диэлектрических слоев, опти- 
ческие толщины которых равны четверти длины волны и металлических слоев, их геометрические толщины не превышают несколько десятков нанометров. На начальной стадии поиска конструкции определяется, какие металлы подходят для использования в структуpax интерференционных систем, которые могут иметь постоянный коэффициент пропускания (отражения) с незначительной разницей в двух компонентах $s$ и $p$ в некоем спектральном диапазоне.

\section{Выбор металлов, входящих в состав неполяризующих интерференционных систем}

Оптические свойства металлов обычно описываются в терминах комплексного показателя преломления: $\tilde{n}=n+i k$, где $n-$ показатель преломления, $k-$ коэффициент поглощения. Для металлов $n$ и $k$ являются функциями длины волны и температуры [11]. Рассмотрим границу раздела между воздухом и металлом в случае, когда излучение падает под некоторым углом $\alpha$ (рис. 1). Амплитудный коэффициент отражения на границе раздела воздух-металл для $s$ - и $p$-поляризаций можно рассчитать по формуле [12]:

$$
r_{s, p}=\frac{\cos \alpha-\tilde{n}_{s, p}}{\cos \alpha+\tilde{n}_{s, p}},
$$

где $\tilde{n}_{s}=\tilde{n} \cos \alpha_{m}$ и $\tilde{n}_{p}=\frac{\cos \alpha_{m}}{\tilde{n}}, \alpha_{m}$ - угол распространения излучения в металле. Угол $\alpha_{m}$ определяется из закона Снеллиуса: $\sin \alpha=n_{m} \sin \alpha_{m}$.

Энергетический коэффициент отражения соответственно $R_{s, p}=\left(\left|r_{s, p}\right|\right)^{2}$.

На рис. 2 представлена спектральная характеристика энергетического коэффициента отражения на границе раздела воздух-металл для $s$ - и $p$-поляризаций. Из анализа зависимости коэффициента отражения $R_{s}{ }^{-}$и $R_{p}$-компонент от угла падение излучения при одной длине волны можно сделать вывод, что значения $R_{s}$ и $R_{p}$ приблизительно равны друг другу при малых углах падения излучения. Поскольку с увеличением угла падения излучения коэффициент отражения для

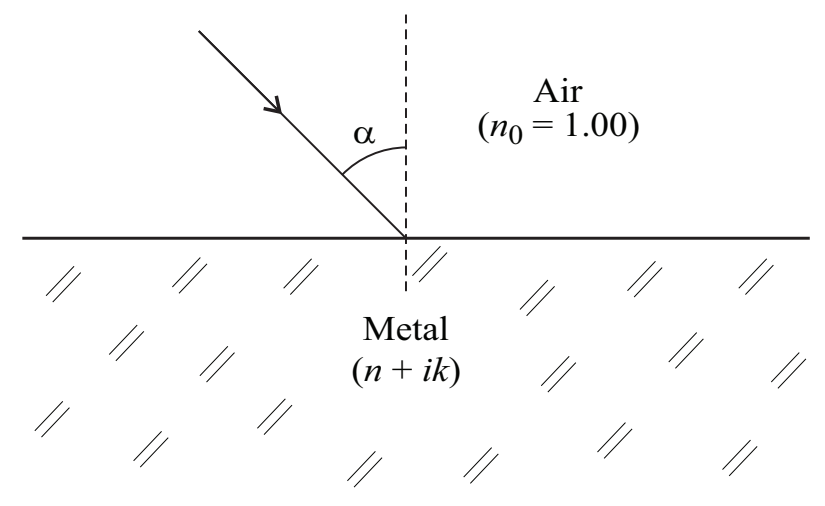

Рис. 1. Граница раздела воздух-металл.

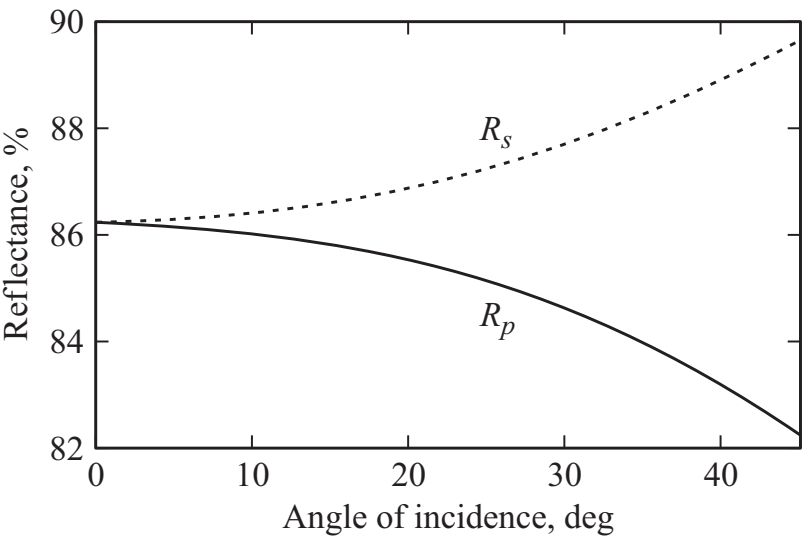

Рис. 2. Зависимости коэффициента отражения $R_{s}$ и $R_{p}$ компонент от угла падения излучения на одной длине волны.

p-поляризации уменьшается, то целесообразно проанализировать возможность увеличения значения энергетического коэффициента отражения для этой компоненты.

Значение энергетического коэффициента отражения $R_{p}$ на границе раздела воздух-металл может быть выражено в приближенной форме следующим обра3ом [13]:

$$
R_{p}=\frac{(n-1 / \cos \alpha)^{2}+k^{2}}{(n+1 / \cos \alpha)^{2}+k^{2}} .
$$

Определим величину $R_{p}$ как функцию угла падения излучения $\alpha$ и найдем минимум этой функции. Минимальное значение $R_{p}$ достигается при условии

$$
\cos \alpha=\frac{1}{\sqrt{n^{2}+k^{2}}}
$$

При этом минимальное значение коэффициента отражения $R_{p}$ будет:

$$
R_{p \min }=\left(\frac{k / n}{1+\sqrt{1+(k / n)^{2}}}\right)^{2} .
$$

Отсюда видно, что значение $R_{p, \min }$ достаточно велико тогда и только тогда, когда величина $k / n$ имеет достаточно большое значение, т.е. когда коэффициент поглощения $k$ металла намного больше его показателя преломления $n$. Этому условию удовлетворяет ряд металлов, таких как серебро (Ag), алюминий (Al) . . [14,15].

\section{Методика поиска конструкций металло-диэлектрических интерференционных систем}

Металло-диэлектрические интерференционные системы состоят из чередующихся металлических и диэлектрических слоев. Диэлектрические слои характеризуются показателями преломления $n_{j}$ и оптическими толщинами $n_{j} d_{j}(j=1,3,5, \ldots)$, а металлические слои - 


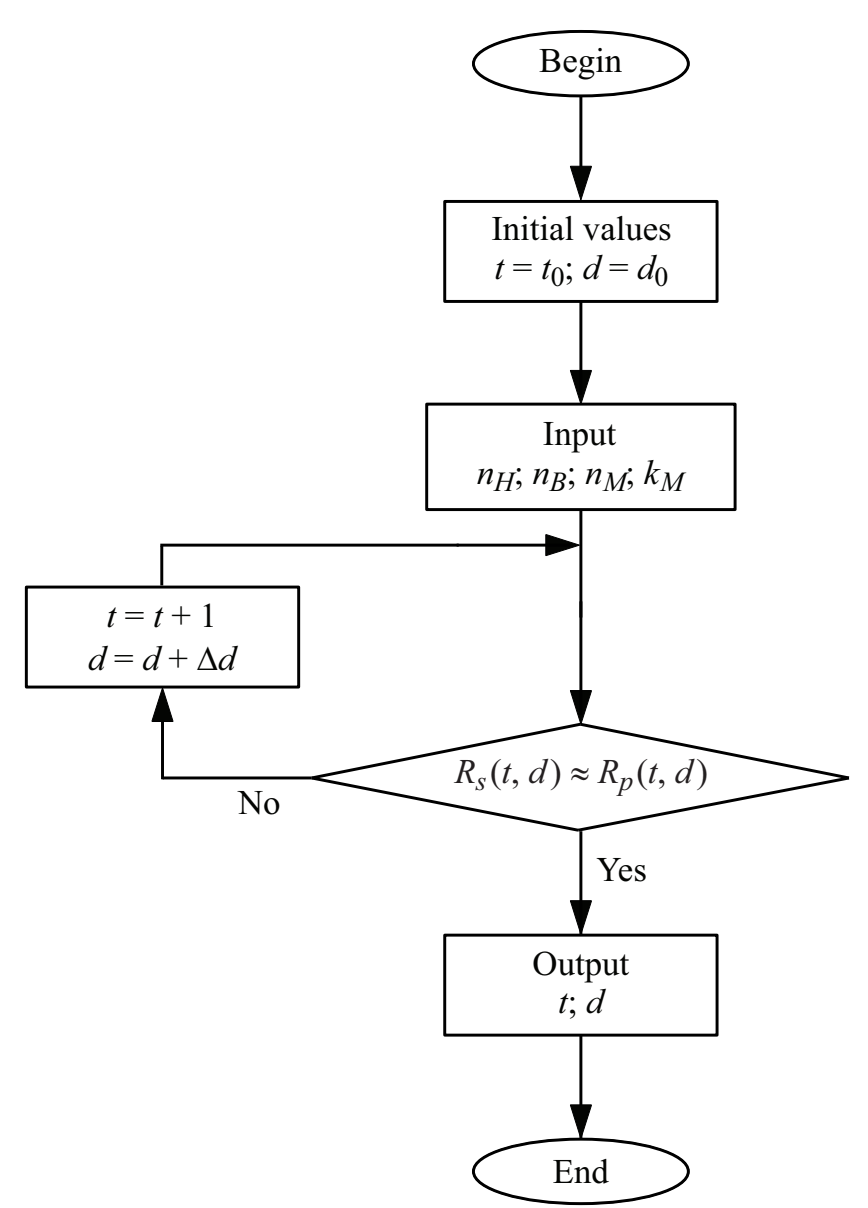

Рис. 3. Процедура поиска параметров структур вида $(H M B)^{t}$.

комплексными показателями преломления $n_{l}+i k_{l}$ и геометрическими толщинами $d_{l}(l=2,4,6, \ldots)$. Первый и последний слои системы обычно изготавливаются из диэлектрических материалов. Рассмотрим интерференционную систему, состоящую из нечетного количества слоев $(N=3,5,7,9, \ldots)$.

Поиск конструкций металло-диэлектрических интерференционных систем (количество слоев, толщина каждого слоя, материал, из которого они изготавливаются) в общем случае является невозможным, так как количество неизвестных параметров в этом случае очень велико. Для простоты рассмотрим частный случай, когда металло-диэлектрические интерференционные системы, изготовленные из диэлектрических слоев, оптические толщины которых равны четверти длины волны $\left(n_{j} d_{j}=\lambda_{0} / 4\right)$, и металлических слоев, их геометрические толщины равны друг другу $\left(d_{1}=d_{l+1}=d\right)$. Рассматриваемая структура имеет вид: $(H M B)^{t}$, где $H$ и $B$ - четвертьволновые диэлектрические слои, изготовленные из материалов с низким и высоким показателями преломления, $M-$ металлические слои, $t-$ повторяемость основной ячейки (HMB).

Процедура поиска параметров рассматриваемой структуры представлена на рис. 3. В начале процедуры вводятся показатели преломления диэлектрических слоев $n_{H}$ и $n_{B}$, значение которых выбраны с учетом существующих пленкообразующих материалов, прозрачных в видимом диапазоне спектра. Показатель преломления и коэффициент поглощения металла выбраны согласно условию, представленному выше. Искомыми параметрами остаются повторяемость $t$ и толщина металлических слоев $d$. Определение их значений осуществляется путем установления зависимости коэффициентов отражения для $s$ - и $p$-поляризаций от повторяемости $t$ и толщины металлических слоев $d: R_{s}(t, d)$ и $R_{p}(t, d)$, основываясь на условии равенства $R_{s}(t, d)=R_{p}(t, d)$, находим удовлетворительные значения искомых параметров $t$ и $d$.

Поиск конструкций металло-диэлектрических интерференционных систем математическим способом является невозможным из-за сложной формы элементов матрицы интерференции металлических слоев, которые обычно представляются в комплексной форме. Графический способ является более простым для поиска конструкции таких покрытий. Графический способ основан на построении графической зависимости спектрального энергетического коэффициента отражения рассматриваемой структуры от геометрической толщины металлического слоя $d$ и повторяемости $t$ для заданной длине волны. Анализ этой зависимости позволяет определить значения повторяемости $t$ и геометрической толщины металлического слоя $d$, при которых энергетические коэффициенты отражения для $s$ - и $p$-поляризаций будут равны.

В качестве примера рассмотрим металло-диэлектрическую интерференционную систему, состоящую из четвертьволновых диэлектрических слоев фторида магния $\mathrm{MgF}_{2}\left(n_{B}=1.38\right)$ и сульфида цинка $\mathrm{ZnS}\left(n_{B}=2.35\right)$. Между ними расположены металлические слои серебpa $\mathrm{Ag}$, имеющие комплексный показатель преломления $n_{\mathrm{Ag}}=0.56+3.35 i$. Эта многослойная система нанесена на поверхность стекла марки К8 $\left(n_{m}=1.52\right)$ (рис. 4). Система может быть представлена в следующем виде:

$$
\text { Воздух }\left|\left(\mathrm{MgF}_{2}|\mathrm{Ag}| \mathrm{ZnS}\right)^{t}\right| \mathrm{K} 8 \text {. }
$$

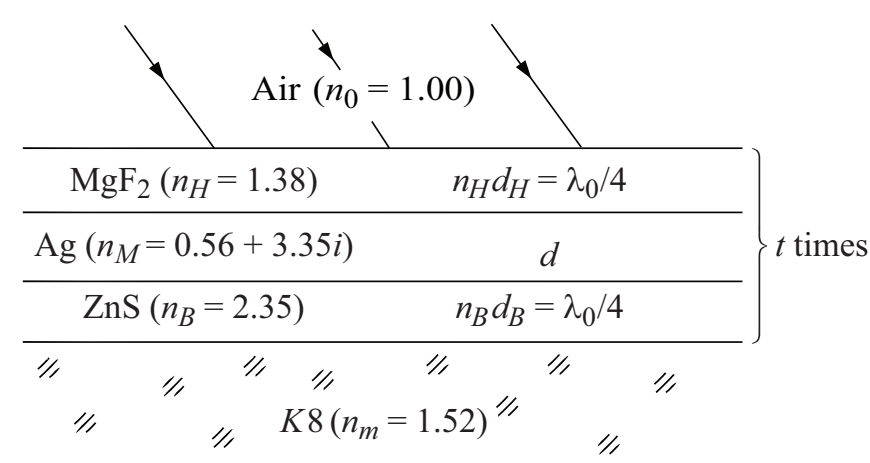

Рис. 4. Металло-диэлектрическая интерференционная система вида Воздух $\left|\left(\mathrm{MgF}_{2}|\mathrm{Ag}| \mathrm{ZnS}\right)^{t}\right| \mathrm{K} 8$ при наклонном падении излучения. 
Для определения значения повторяемости $t$ воспользуемся процедурой, показанной на рис. 3 , для чего используем программy MathLab, при условии, что геометрическая толщина металлических слоев выбрана из набора некоторых заданных значений. В результате вычисления получено некое значение повторяемости $t=6$, т. е. Рассматриваемая система состоит из 18 слоев. Определение геометрической толщины металлических слоев графическим способом выполняется построением зависимости коэффициентов отражения для $s$ - и $p$-поляризаций от длины волны $\lambda$ и геометрической толщины $d$. На рис. 5, $a$ представлено распределение коэффициентов отражения для $s$ - и $p$-поляризаций в диапазоне длины волны от 460 до $580 \mathrm{~nm}$ и геометрической толщины от 20 до $60 \mathrm{~nm}$. На этом рисунке наблюдаются зоны, в которых значения энергетических коэффициентов отражения $R_{s}$ и $R_{p}$ будут приближёнными (зоны вокруг кривых 82\%). На рис. $5, b$ представлены зависимости коэффициентов отражения $R_{s}$ и $R_{p}$ от геометрической толщины металлических слоев при длине волны $550 \mathrm{~nm}$. Из этого рисунка можно определить значение геометрической толщины металлических сло-
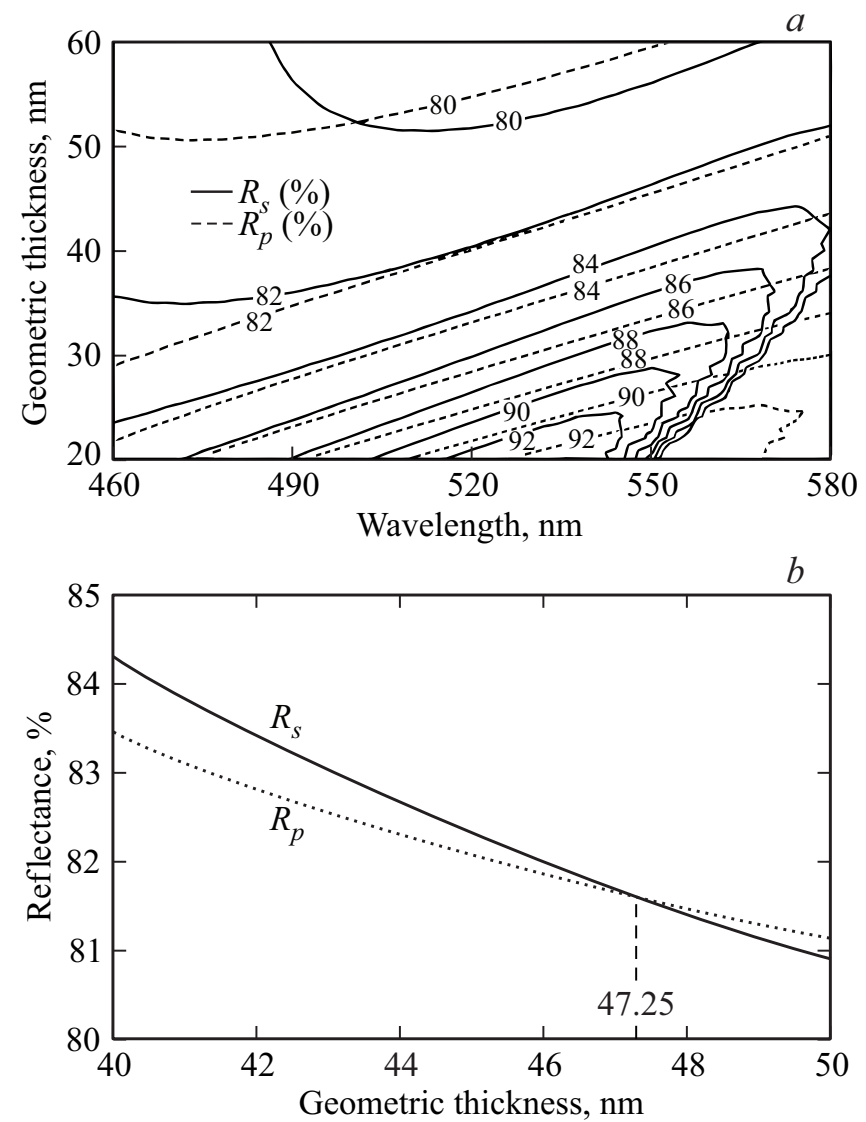

Рис. 5. Распределение коэффициентов отражения системы Воздух $\left|\left(\mathrm{MgF}_{2}|\mathrm{Ag}| \mathrm{ZnS}\right)^{6}\right| \mathrm{K} 8$ для $s$ - и $p$-поляризаций при угле падения излучения $45^{\circ}(a)$. Зависимость коэффициентов отражения системы Воздух $\left|\left(\mathrm{MgF}_{2}|\mathrm{Ag}| \mathrm{ZnS}\right)^{6}\right| \mathrm{K} 8$ от геометрической толщины металлических слоев для $s$ - и $p$ - поляризаций на длине волны $550 \mathrm{~nm}$ и при угле падения излучения $45^{\circ}(b)$.

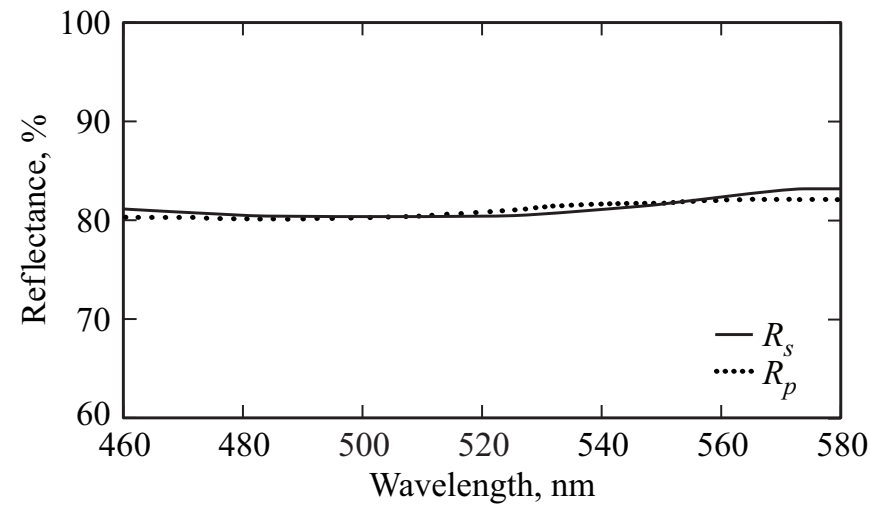

Рис. 6. Спектральные зависимости коэффициентов отражения. Воздух $\left|\left(\mathrm{MgF}_{2}|\mathrm{Ag}| \mathrm{ZnS}\right)^{6}\right| \mathrm{K} 8$ для $s$ - и $p$-поляризаций при угле падения излучения $45^{\circ}$.

ев, при котором энергетические коэффициенты отражения $s$ - и $p$-поляризаций равны друг другу. Это значение равно $47.25 \mathrm{~nm}$. В результате графическим способом определены все параметры слоев, входящих в состав рассматриваемой системы.

На рис. 6 представлены спектральные характеристики коэффициентов отражения полученной интерференционной системы для $s$ - и $p$-поляризаций в диапазоне длины волн $460-580 \mathrm{~nm}$ при угле падения излучения $45^{\circ}$. На этом рисунке видно, что расхождение между спектральными характеристиками для $s$ - и $p$-поляризаций в рассматриваемом диапазоне спектра отраженного излучения достаточно незначительное. Разница между интегральными коэффициентами отражения для $s$ - и $p$-поляризаций в этом диапазоне спектра составляет $0.05 \%$.

\section{Заключение}

$\mathrm{B}$ настоящей работе приведена методика поиска конструкции неполяризующих интерференционных покрытий, в состав которых входят слои, изготовленные из металлических материалов. Также представлена методика выбора металла, из которого выполнены один или несколько слоев, для чего обеспечивалось малое расхождение между спектральными характеристиками энергетического коэффициента отражения (пропускания) для $s$ - и $p$-поляризаций. Полученная структура покрытий достаточно простая в реализации из-за небольшого количества слоев, причем оптические толщины диэлектрических слоев равны четверти длины волны и геометрические толщины металлических слоев равны между собой. В результате анализа спектральных характеристик коэффициента отражения разработанной конструкции видно, что расхождение между спектральными характеристиками для $s$ - и $p$-поляризаций является достаточно незначительным в рассматриваемом диапазоне спектра. 


\section{Финансирование работы}

Работа выполнена при финансовой поддержке Министерства образования и науки Российской Федерации (Проект 16.1651.2017/4.6).

\section{Конфликт интересов}

Авторы заявляют, что у них нет конфликта интересов.

\section{Список литературы}

[1] Baumeister P.W. Optical Coating Technology. USA: SPIE, 2004. 840 p.

[2] Costich V.R. // Appl. Opt. 1970. V. 9. N 4. P. 866-870.

[3] Baumeister P. // Opt. Act. 1961. V. 8. N 2. P. 105-119.

[4] Thelen A. // Appl. Opt. 1976. V. 15. N 12. P. 2983-2985.

[5] Wang W., Xiong S., Zhang Y. // Appl. Opt. 2007. V. 46. N 16. P. 3185-3191.

[6] Rolf E.H., Karl H.G. Handbook of Optical Properties: Thin Films for Optical Coatings. V. 1. USA: CRC Press, 1995. $384 \mathrm{p}$.

[7] Macleod A.H. Thin-Film Optical Filters, Fourth Edition. USA: CRC Press, 2010. 800 p.

[8] Jin H.S., Chun Y.G., Zheng P.W. // Appl. Opt. 2009. V. 48. N 18. P. 3385-3390.

[9] Hongji Q., Ruijin H., Kui Y., Jianda S., Zhengxiu F. // Appl. Opt. 2005. V. 44. N 12. P. 2343-2348.

[10] Shkliarevskii I.N., Avdeenko A.A. // Opt. Spectrosc. 1959. N 6. P. 439-443.

[11] Ефимов А.М. Оптические свойства материалов и механизмы их формирования. Спб.: СПБГУИТМО, 2008.103 с.

[12] Губанова Л.А., Путилин Э.С. Оптические покрытия. Учебник для вузов. Спб.: Лань, 2016. 286 с.

[13] Goldstein D.H. Polarized Light, 2nd ed. Marcel Dekker, 2003. P. 486.

[14] Schulz L.G. // J. Opt. Soc. Am. 1954. V. 44. P. 357-362.

[15] Schulz L.G., Tangherlini F.R. // J. Opt. Soc. Am. 1954. V. 4. P. 362-367. 